Article

\title{
Not ready to Kwit? A mobile app preparation program for smoking cessation: assessing feasibility in ecological context
}

\author{
Luz Bustamante 1-2*, Mickael Ehrminger ${ }^{2}$ and Lucia Romo ${ }^{1,3}$ \\ 1 Laboratoire EA 4430-CliPsyd, Department of Psychology, University of Paris Nanterre, Nanterre, Ile-de- \\ France, France; \\ 2 Research and development department, Kwit SAS ; mickael@kwit.app \\ 3 Hopital Raymond-Poincaré, Assistance Publique Hopitaux de Paris; romodes@parisnanterre.fr \\ * Correspondence: luz.bustamanteperez@parisnanterre.fr
}

\begin{abstract}
Aim: The main purpose of this feasibility study was to estimate compliance and acceptability rate of a 9-Steps mobile app preparation program. Subsequently, to assess its impact regarding attitudes towards smoking cessation. Methods: The program was only available on versions above iOS 13.3. The ecological study collected user's data via the app from 04/07/2021 to 28/07/2021. Results: A total of 2331 ecological users completed onboarding; 1926 users (73.9\%) completed the step 0 and only 265 users continued in step 1 . On average, participants actively engaged with intra-steps activities (85,1\%). Most users were highly satisfied at the end of each step. On average $86 \%$ found the content of each step comprehensive and relevant. Mann-Whitney tests demonstrated positive changes in attitudes toward smoking cessation on the less confident users: willing ( 3 to $5, \mathrm{p}<.05$ ); confidence ( 3 to $5, \mathrm{p}<.001$ ) and readiness to quit $(3$ to $5, \mathrm{p}<.001)$. Conclusions: This ecological study corroborates the expected dropout between first use and regular use of health mobile apps. However, engagement and satisfaction rates support the feasibility and acceptability of a 9-step preparation program. Additional research is needed to better understand determinants of regular use of mobile apps and its impact on the target behavior.
\end{abstract}

Keywords: Smoking cessation; digital therapeutics; behavior change techniques; feasibility; engagement, preparation phase; attitudes toward change, functionality

\section{Introduction}

Although the smoking rate is declining worldwide (from $22.7 \%$ in 2007 to $17.5 \%$ in 2019), more than 1 billion people continue to smoke [1]. Support is needed to help $60 \%$ of smokers intending to quit and $40 \%$ of those trying to quit during the year [1]. In addition to the already known health harms caused by smoking, such as cancer [2] and cardiorespiratory diseases $[2,3]$, smoking has been reported to be an independent risk factor for COVID-19 progression, including mortality [4].

There are two main categories of effective treatments for smoking cessation: pharmacotherapy and psychological interventions [5], although according to WHO, only $18 \%$ of the population would have access to effective treatments [6]. Combining pharmacotherapy with behavioral intervention has been shown to increase the odds of remaining smoke-free at six months by $70 \%$ to $100 \%$ compared to usual care or minimal intervention [7]. The inclusion of several therapeutic principles is, in fact, one of the advantages of digital health intervention like m-health apps [8-10]. It allows the user to foster a sense of responsibility and commitment to their health by, for example, tracking their nicotinic 
substitutes and, at the same time, having access to cognitive and behavioral treatment based on psychologic principles that have already been proven to be effective.

Another advantage of mobile applications is the ability integrating gamification principles or playful elements [11], such as points and rewards accumulated according to the achievement of specific objectives or levels or sharing one's successes with friends or on social networks.

The main interest of using this gamification strategy in an application is to reinforce the user's engagement in the long term by making the activities more fun and enjoyable [12-14]. The ability to share results also develops a certain sense of challenge that motivates users to go further, increases their self-esteem and pride in their accomplishments[15]. It is also known that playful elements promote learning new skills and knowledge $[14,16]$. This approach increases the chances of successful cessation as shown in a study using Kwit [17]. Also, another study showed that mobile applications, increase user's motivation and sense of self-efficacy of users in the cessation process, which increases the chances of success [18]. Indeed, motivation and a sense of self-efficacy (confidence in one's abilities) are important determinants of successful cessation[19-22].

Mobile apps can be an essential lever to promote smoking cessation, and to access effective treatments as almost $70 \%$ of the world's population own a smartphone and internet access [23], and the use of mobile apps to quit smoking would increase the probability of maintaining smoking cessation by almost $25 \%$ [24]. Nevertheless, even if an app includes all the therapeutic guidelines, it cannot be effective if it is not used regularly [24]. As in any treatment, "adherence to therapies is an important determinant of treatment success"[25]. Indeed, understanding how users engage in their own health treatment and choose to interact regularly with apps is a current challenge for mobile apps in general [26] and m-health apps [27]. Therefore, it is essential to identify and assess the main factors that favor the use mobile apps for smoking cessation. To this end, the various research methods should ensure ecological validity and ensure that the behavioral responses obtained represent the natural behaviors of people who want to quit smoking with the help of a mobile app [27].

\subsection{Present Study}

The present study is part of a larger project that aims to identify the key factors that determine the regular use of mobile health apps and their use on the targeted behavior. For the project, we developed a 9-step mobile app preparation program for smoking cessation. Moreover, the main objective of this study is to determine whether the program is ready to be used and tested in a real-world context. We want to anticipate major technical issues that may affect the implementation of the leading project and explore three central questions:

1) How many "organic users" (which we define as "users who voluntarily and independently download the application, without paid advertising campaign") are willing to prepare their smoking cessation process with a mobile app?

2) How many "organic users" will engage with the program, knowing that a big dropout is expected between the first and the second use of any mobile app [26]?

3) How relevant is the evidence-based content proposed to users' experience? Do they think the program content is clear and relevant to their smoking cessation process? Besides, users' perception of content understandability and relevance may explain a loss of engagement towards the program [28]

Subsequently, we want to explore how the program affects users' attitudes toward smoking cessation. We expect the completion of the program will have a positive impact as it has been the case in other interventions [24]. 


\section{Materials and Methods}

\subsection{Study design}

This prospective and observational study was conducted in an ecological context from $07 / 04 / 2021$ to $07 / 28 / 2021$. For this feasibility study the program was only available on versions above iOS 13.3. Organic users downloaded Kwit mobile app, and after finishing the onboarding process leading to the preparation program, user's data started to be collected. No compensation was proposed to the users for participating in this study.

Participants were aged of 18 years and above, French or English speaking, owned and used a compatible smartphone (iOS 13.3 and above) with regular internet service to access the program. Participants created an account that pseudonymizes their personal information. Only data pre-required for the study was exported to be analyzed, as detailed thereafter.

\subsubsection{Consent and Ethical Approval}

This feasibility study is part of a prospective study which aims to identify key determinants of smoking cessation mobile apps use among smokers seeking to quit.

The protocol study has been validated by the local French Ethical Committee (SouthEast) on March the $25^{\text {th }}, 2021$. The Research Identification Number is 2020-A02733-36, and the reference of the committee is CPP 20.10.02.44945. This study is also in accordance with the ethical principles of the Declaration of Helsinki.

\subsubsection{Onboarding}

The onboarding journey (see Supplementary Figure 1) consists in six screens leading to an account creation. Users are asked for a pseudonym, the stage of change they are at, current tobacco habits (number of daily cigarettes smoked, number of cigarettes per pack, and its price). Participants who created their account and identified themselves as being in preparation stage (from Prochaska and DiClemente model of change theory) were proposed to pay for the premium version, and if they didn't want to access the paid premium version, they had direct access to the free version of the program.

The free version of the program is composed of four steps: Step 0, Step1, Step 2 and the final Step 8. Once free users have finished the step 8, they have access to the free version of the current Kwit app.

At any time, users could acquire the premium version giving them access to the complete 9-step program.

\subsubsection{Kwit preparation program}

Kwit preparation program is the result of two years of $R \& D$, as part of a $\mathrm{PhD}$ thesis, in collaboration with the UPN, University of Paris-Nanterre. The program was created by a team composed by a user experience designer, three software developers with at least 10 years of experience, a researcher in public health and clinical research, as well as a cognitive and behavioral psychologist and PhD student.

Users had access to a pilot version of the program with all steps described in Table 1. Within each step, an informative screen presented the main theme to be treated, followed by a list of activities to be unblocked (see Supplementary Figure 2). At the end of the step user are brought back to the dashboard menu. Each step's activity was presented as follows: a first informative screen, followed by the interactive screens' activity, then with personalized feedback and ended back on the dashboard screen. The version of the program used in this study does not have a gamified layer in which users gain points at the 
end of each activity, nor educational reading contents which will be published in further versions.

The 9-step Kwit's preparation program has been developed for commercialization and designed for direct delivery as a mobile app intervention on Apple and Google Stores. This program is based primarily on cognitive and behavioral therapy for smoking cessation and inspired on dialectical and behavioral therapy, motivational interviewing, and self-determination theory.

The detailed content of each step is described as follows: the step 0 is about becoming aware of one's motivations to commit to the change process (smoking cessation). The step 1 is about learning to observe oneself and the unwanted behavior; to create a baseline of what needs to be changed and easily acknowledge the evolution. To do so, in step 2 the program highlights the importance of understanding what prompts the unwanted behavior and start advising to use new behavioral strategies. The step 3 is about describing the unwanted behavior according to its short-term benefits (pleasure or relief). The fourth step invites users to experience the change they feel able to make on the 24-hours period. The three next steps are about learning new strategies to cope with the three dependence types. The final step invites users to define a quit date and acknowledge the cognitive barriers to quit. The whole program encourages being kind to oneself when slips and relapses occur and celebrate each small step towards the desired change. Table 2 details this behavioral change program according to Michie's taxonomy [29].

Table 1. Overview of Behavioral and Cognitive Techniques and goals presented in the 9-step Kwit's preparation program.

\begin{tabular}{|c|c|c|c|}
\hline Name of Step & Main goal & BCTs label $^{*}$ & Definition \\
\hline $\begin{array}{l}\text { Step } 0 \text { (S0): } \\
\text { Defining what is } \\
\text { important }\end{array}$ & $\begin{array}{l}\text { Identify personal } \\
\text { values and moti- } \\
\text { vations to quit } \\
\text { smoking }\end{array}$ & Commitment & $\begin{array}{l}\text { Ask the person to affirm or reaffirm statements indicat- } \\
\text { ing commitment to change the behavior data. }\end{array}$ \\
\hline $\begin{array}{l}\text { Step } 1 \text { (S1): } \\
\text { Adopting an ob- } \\
\text { server attitude }\end{array}$ & $\begin{array}{l}\text { Observe current } \\
\text { consumption } \\
\text { habits through } \\
\text { the recording of } \\
\text { cravings during a } \\
\text { typical day. }\end{array}$ & $\begin{array}{l}\text { Instruction on how } \\
\text { to perform a be- } \\
\text { havior } \\
\text { Self-monitoring of } \\
\text { the behavior: }\end{array}$ & $\begin{array}{l}\text { Advise or agree on how to perform the behavior in the } \\
\text { app: craving log and meditation exercise } \\
\text { Establish a method for the person to monitor and record } \\
\text { their behavior as part of a behavior change strategy. }\end{array}$ \\
\hline \multirow{3}{*}{$\begin{array}{l}\text { Step } 2(\mathrm{~S} 2) \text { : } \\
\text { Understanding } \\
\text { my past journey }\end{array}$} & \multirow{3}{*}{$\begin{array}{l}\text { Observe of past } \\
\text { consumption } \\
\text { habits }\end{array}$} & $\begin{array}{l}\text { Information about } \\
\text { antecedents }\end{array}$ & $\begin{array}{l}\text { Provide information about antecedents that reliably pre- } \\
\text { dict performance on the behavior. }\end{array}$ \\
\hline & & Re-attribution & $\begin{array}{l}\text { Elicit perceived causes of behavior and suggest alterna- } \\
\text { tive explanations. }\end{array}$ \\
\hline & & $\begin{array}{l}\text { Restructuring the } \\
\text { physical/social en- } \\
\text { vironment }\end{array}$ & $\begin{array}{c}\text { Advise to change the physical environment to facilitate } \\
\text { performance of the wanted behavior or create barriers to } \\
\text { the unwanted behavior }\end{array}$ \\
\hline $\begin{array}{l}\text { Step } 3(\mathrm{~S} 3) \text { : } \\
\text { Acting with } \\
\text { awareness }\end{array}$ & $\begin{array}{l}\text { Classify of crav- } \\
\text { ings between }\end{array}$ & $\begin{array}{l}\text { Instruction on how } \\
\text { to perform a be- } \\
\text { havior }\end{array}$ & $\begin{array}{l}\text { Advise or agree on how to perform the behavior in the } \\
\text { app: craving classification. }\end{array}$ \\
\hline
\end{tabular}




\begin{tabular}{|c|c|c|}
\hline $\begin{array}{l}\text { anchored and re- } \\
\text { flex cravings. }\end{array}$ & $\begin{array}{l}\text { Feedback on be- } \\
\text { havior }\end{array}$ & $\begin{array}{l}\text { Monitor and provide informative or evaluative feedback } \\
\text { on performance of the behavior (frequency, duration, in- } \\
\text { tensity, context). }\end{array}$ \\
\hline
\end{tabular}

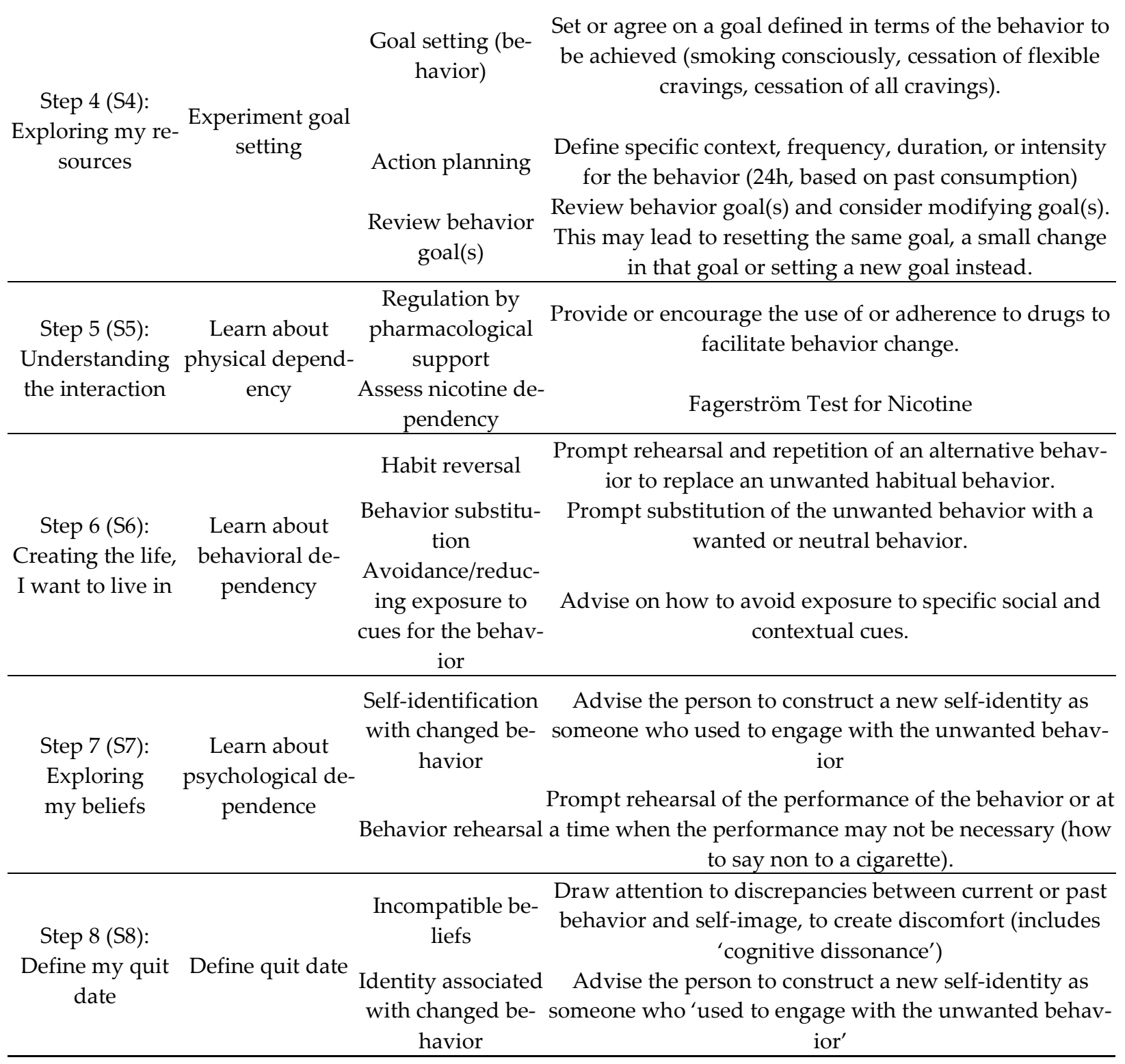

\subsection{Outcome Variables and Measurement}

Four different types of response categories were used: Visual Analogue Scale (VAS slider) (see Figure 3a), multiple-choice questionnaires (see Figure 3b) and Event Assessment ("a method of data collection whereby a recording is made each time a predefined event occurs") [30]. Visual Analogue Scale was used to measure motivation level. Multiple-choice questionnaires were used to measure satisfaction, motives to quit and motivation type. And Event Assessment was required to measure all engagement metrics.

\subsubsection{Engagement}

User's engagement was assessed through four metrics. 
- Program completion and follow-up rate: the proportion of users who complete the program from first use (S0) to the final in-app activity (S8) and, who independently continue logging on the app upon competition of the program.

- Inter-step completion rate: the proportion of users who moved from one step to their immediate next step. Two metrics were measured, as recommended [31], the intention-to-treat (ITT) completion rate, which refers to the proportion of the initial sample accessing at each step, and the per-protocol (PP) completion rate, which refers to the proportion of users from one step passing through to the next step.

- Intra-step completion rate: the proportion of users who accessed and finished each activity proposed inside each step.

- Time completion: the days required for users to complete the program.

\subsubsection{Satisfaction toward app content}

Users answered a survey to assess understandability and relevance of each step content. The question was presented as follows: "You have finished Step X. How would you define it?", with those three options: "Understandable and relevant; Understandable but irrelevant; Not understandable and irrelevant".

\subsubsection{Motivation}

Three self-reported metrics of motivation were measured: motives to quit, motivation type, motivation level.

Motives to quit smoking was assessed through users' in-app selection of one of five proposed reasons in the app: health, well-being, economy, family, and baby project.

Motivation type was assessed through an ad hoc 18-items questionnaire based on self-determination theory. The questionnaire is validated (article in preparation). The items are divided into six subscales corresponding to the different types of motivation: Amotivation, External, Identified, Integrated, Introjected, Intrinsic. A Mixed motivation profile was defined when no clear trend emerged from the individual's results. The participants indicate on a Likert-type scale ranging from 1 (Does not correspond at all) to 5 (Corresponds exactly), to what extent they agree or disagree with each of the items.

Motivation level was assessed through a visual analogue scale of three questions measuring willingness, ability, and readiness to quit. Users could move the cue from 1 (the minimum score) to 10 (the highest) for each of the following questions: "To what extent is change a priority for you right now?", "To what extent are you confident in your ability to change right now?", "To what extent do you feel ready to take action?".

\subsection{Data collection and Statistical Analyses}

Participants created an account that pseudonymized their personal information. Data were collected electronically through participant input throughout the preparation program (downloaded directly from users). Pseudonymized study data were imported into a secure database (Firebase, Google Cloud Services) and only previously defined variables were exported to be analyzed on R-Studio version 1.4.1106.

Descriptive analyses were used to analyze engagement, satisfaction, and motivation measures. The Mann-Whitney test was used to determine if there was a significant impact of the program on motivation status and the Chi-square tests for comparing frequencies. 


\section{Results}

A total of 2331 users started the preparation program, 61\% $(n=1379)$ had the French version and 39\% $(n=952)$ the English version. 2149 were free users and 182 were premium users.

\subsection{Engagement toward app use}

Table 2 presents the completion rate evolution for each activity of the preparation program, in terms of: intra-step completion rate and inter step completion rate by intention-to-treat and per protocol.

Table 2. Quantity and proportion of participants starting and completing each step by those who onboarded $(n=2331)$.

\begin{tabular}{|c|c|c|c|c|c|c|}
\hline Step & Activity & Started & Completed & $\begin{array}{l}\text { Intra-step } \\
\text { completion }\end{array}$ & $\begin{array}{l}\text { ITT inter- } \\
\text { step } \\
\text { completion }\end{array}$ & $\begin{array}{c}\text { PP } \\
\text { inter-step } \\
\text { completion }\end{array}$ \\
\hline 0 & 1 & 2331 & 2328 & \multirow{4}{*}{$85 \%$} & & \\
\hline 0 & 2 & 2259 & 2248 & & & \\
\hline 0 & 3 & 2027 & 2024 & & & \\
\hline 0 & 4 & 1998 & 1974 & & & \\
\hline 1 & 1 & 265 & 261 & \multirow{2}{*}{$88 \%$} & \multirow{2}{*}{$11.4 \%$} & \multirow{2}{*}{$13 \%$} \\
\hline 1 & 2 & 236 & 232 & & & \\
\hline 2 & 1 & 195 & 195 & \multirow{2}{*}{$94 \%$} & \multirow{2}{*}{$8.4 \%$} & \multirow{2}{*}{$84 \%$} \\
\hline 2 & 2 & 184 & 184 & & & \\
\hline $3^{1}$ & 1 & 46 & 46 & \multirow{3}{*}{$83 \%$} & \multirow{3}{*}{$2.0 \%$} & \multirow{3}{*}{$\mathrm{P}=100 \%$} \\
\hline 3 & 2 & 42 & 42 & & & \\
\hline 3 & 3 & 38 & 38 & & & \\
\hline 4 & 1 & 31 & 31 & \multirow{4}{*}{$42 \%$} & \multirow{4}{*}{$1.3 \%$} & \multirow{4}{*}{$82 \%$} \\
\hline 4 & 2 & 29 & 29 & & & \\
\hline 4 & 3 & 15 & 15 & & & \\
\hline 4 & 4 & 13 & 13 & & & \\
\hline 5 & 1 & 11 & 10 & $91 \%$ & $0.5 \%$ & $85 \%$ \\
\hline 6 & 1 & 9 & 9 & $100 \%$ & $0.4 \%$ & $90 \%$ \\
\hline 7 & 1 & 9 & 9 & \multirow{2}{*}{$100 \%$} & \multirow{2}{*}{$0.4 \%$} & \multirow{2}{*}{$100 \%$} \\
\hline 7 & 2 & 9 & 9 & & & \\
\hline $8^{2}$ & 1 & 59 & 57 & \multirow{3}{*}{$92 \%$} & $2.5 \%$ & $55 \%$ \\
\hline 8 & 2 & 56 & 56 & & $\mathrm{~F}=2.2 \%$ & $\mathrm{~F}=42 \%$ \\
\hline 8 & 3 & 54 & 54 & & $\mathrm{P}=3.3 \%$ & $\mathrm{P}=67 \%$ \\
\hline \multicolumn{2}{|c|}{ Maintain use ${ }^{3}$} & 16 & - & - & $0.7 \%$ & $29 \%$ \\
\hline
\end{tabular}

${ }^{1}$ Premium status ( $\mathrm{P}, \mathrm{n}=46$ at the beginning of S3) was needed to access S3 through S7.

${ }^{2}$ Users continuing with the free version (F) directly access the final step (S8) after finishing S2.

${ }^{3}$ Users who continue using the application after the completion of the final step (S8).

$\mathrm{F}=$ free users; $\mathrm{P}=$ premium users 


\subsubsection{Program completion and follow-up rate:}

Of the initial sample $2.3 \%(n=54)$ complete the final activity (S8) of the program with no significant difference $(p=0.31)$ between free $(2.2 \%)$ and premium users $(3.3 \%)$. $29.6 \%(n=16)$ of the users who complete the final step (S8) of the program access the application in the following 10 days. This usage was limited to one connection.

\subsubsection{Inter-step completion rate:}

Two dropout points were observed. The first one between step 0 and step 1 and the second one between step 2 to step 3. At the first dropout point, $13 \%(n=265)$ of user's went on with the program (PP), representing $11.4 \%$ of the initial sample (ITT). Regarding the second dropout point, only $25 \%(n=46)$ of the users still in the program in the previous step went on, meaning they converted to premium status, representing this time $2 \%$ of the initial sample.

As a reminder, the free version of the app allows users to access four steps: S0, S1, S2 and S8. That's why at step 3, we observed a large decrease in the number of users (the free users don't have access to it), while at step 8 we observed an increased number of users ( $n=59$ free and premium users) compared with the number of users finishing the step 7 ( $\mathrm{n}=9$ premium users only).

The other transitions between steps indicate good engagement by keeping $82 \%$ to $100 \%$ of users from the last activity of a step to the first activity of the follow step.

\subsubsection{Intra-step completion rate:}

The intra-step completion rate varied between $83 \%$ to $100 \%$, apart from step 4 where the completion rate was $42 \%$.

\subsubsection{Completion time (in days) of the program.}

Regardless of the different number of free vs. premium steps, users of the free version completed their 4-step program in a median of 2 days $(\mathrm{Q} 1=1 / \mathrm{Q} 3=3)$ while users of the premium version completed their 9-step program in a median of 4 days $(\mathrm{Q} 1=3 / \mathrm{Q} 3=5)$. When considering only the four shared steps (S0, S1, S2 and S8), results showed no significant difference $(P=0,08)$ between users of the free $($ median $=2, \mathrm{Q} 1=1 / \mathrm{Q} 3=3)$ or the premium version (median $=2, \mathrm{Q} 1=1 / \mathrm{Q} 3=2)$ with a sample median of 2 days $(\mathrm{Q} 1=1 /$ Q3 = 3).

\subsection{Sastisfaction with app content}

Most users reported high satisfaction levels at the end of each step. On average 85.9\% $(\mathrm{SD}=7.3)$ found the content of each step understandable and relevant. A minority of users found the content understandable but irrelevant $(\mathrm{M}=13.3 \%, \mathrm{SD}=6.4)$. S1 received the lowest score of satisfaction with $71 \%$ founding it relevant and understandable and $27 \%$ finding it understandable but irrelevant.

\subsection{Motivation and attitudes toward changing behaviors}

\subsubsection{Reasons to quit}

The main motivations to quit among users was distributed as follows (unique choice): health $(50 \%)$, economy (19\%), well-being $(18 \%)$, family $(7 \%)$ and baby project $(5 \%)$. No marked difference was observed between men and women $(p=0,1)$. Free $(20 \%)$ and premium $(8 \%)$ users only had significantly different attitudes toward quitting smoking to save money $(p=0.02)$. 


\subsubsection{Motivation type}

Table 3 presents the percentage of every motivation type assessed by our questionnaire based on self-determination theory. The most represented motivation type was extrinsic motivation $(58.5 \%)$, followed by intrinsic motivation $(24.8 \%)$, mixed motivation $(12.7 \%)$ and amotivation $(4.1 \%)$. Among the extrinsic motivation types, the most frequently observed was the integrated motivation ( $34.8 \%$ of extrinsically motivated users).

Table 3. Percentage of motivation type among total users and free and premium version users.

\begin{tabular}{lccc}
\hline & Total & Free & Premium \\
\hline Amotivation & 4.1 & 4.3 & 1.8 \\
Extrinsic Motivation & 58.5 & 58.7 & 55.1 \\
$\quad$ External & 11.3 & 11.3 & 11.5 \\
$\quad$ Introjected & 4.5 & 4.5 & 3.6 \\
$\quad$ Identified & 7.9 & 7.7 & 9.7 \\
$\quad$ Integrated & 34.8 & 35.2 & 30.3 \\
Intrinsic & 24.8 & 24.2 & 30.9 \\
Mixed & 12.7 & 12.7 & 12.1 \\
\hline
\end{tabular}

No significant difference was observed between users of free and premium version $(p=0.23)$. Nor was there any difference between users who completed the program and the ones who abandoned it en route $(p=0.9)$.

\subsubsection{Motivation level.}

Figures 1 and 2 present the evolution of willingness, ability, and readiness to quit from baseline (S0) to end of program (S8) among all users, and among the sub-sample of users with initial levels of willingness, ability, and readiness lower than 5/10.

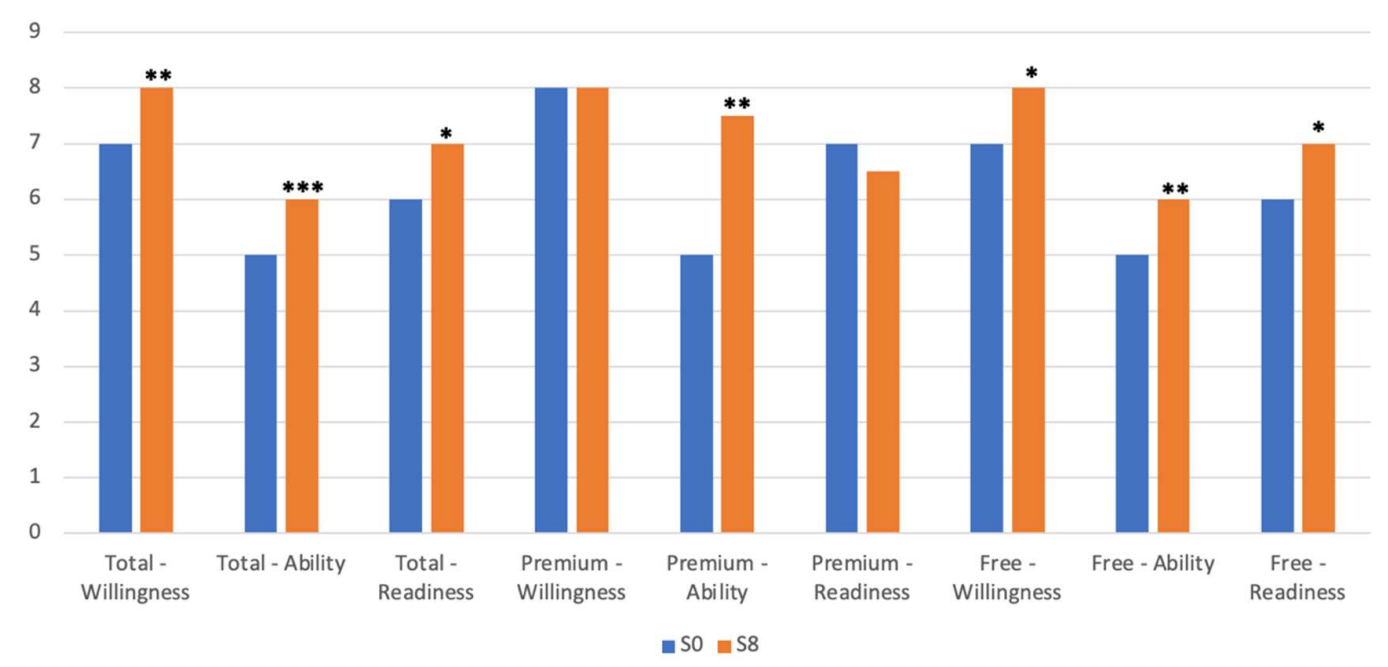

Figure 1. Evolution of the motivational indices between S0 and S8 in the total sample, in the premium and the free sub-samples. Levels of significance of the difference between $\mathrm{S} 8$ and S0: ${ }^{*} \mathrm{p}<0.05,{ }^{* *} \mathrm{p}<0.01,{ }^{* * *} \mathrm{p}<0.001$

Using a Mann-Whitney test, a moderate significant improvement is observed for all three metrics between baseline and the end of the program for the entire sample: willingness $(p=0.003)$, ability $(p<0.001)$, readiness $(p=0.02)$. At Step 0 premium users reported 
significantly higher willingness $(p<0.001)$, ability $(p=0.04)$, and readiness $(p=0.004)$ to quit than free users.

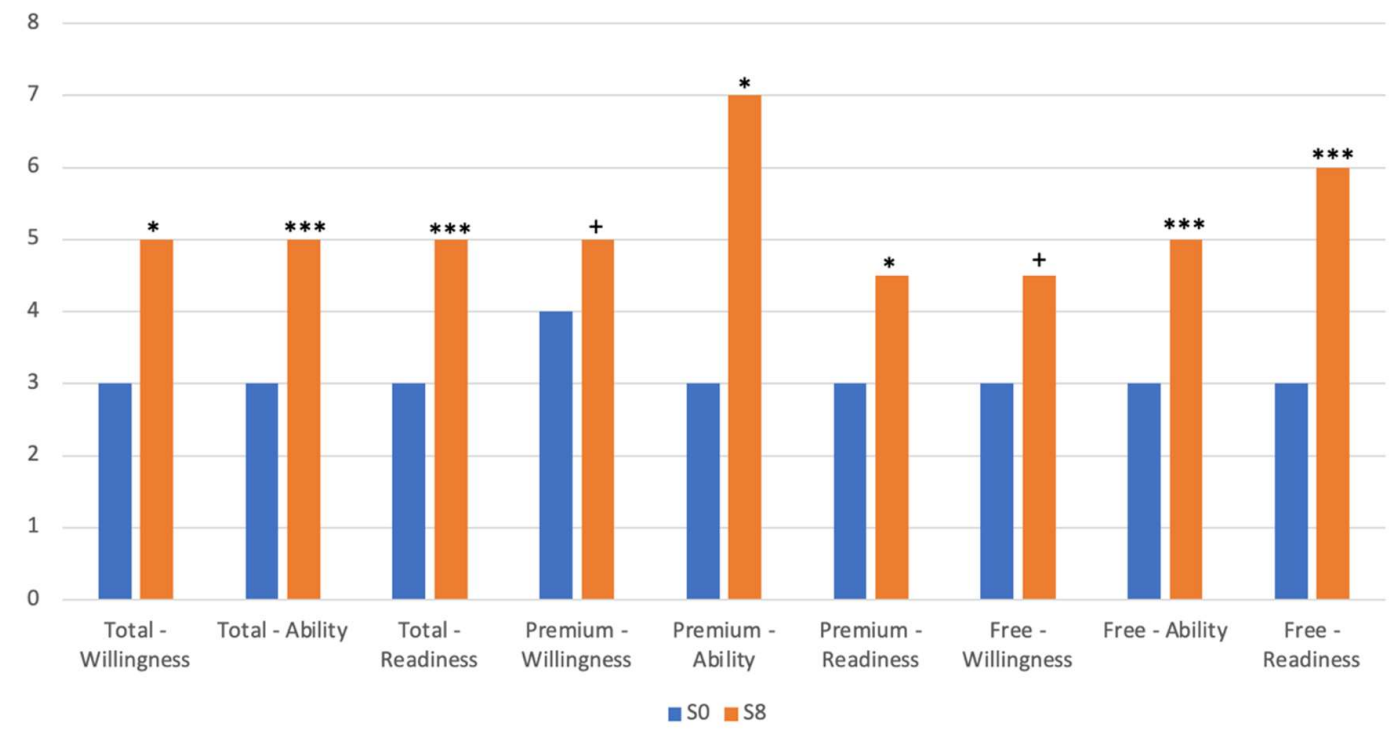

Figure 2. Evolution of the motivational indices between $S 0$ and $S 8$ in the sub-samples with motivational indices lower than $5 / 10$ at S0. Levels of significance of the difference between S8 and S0: $+\mathrm{p} \sim 0.05,{ }^{*} \mathrm{p}<0.05,{ }^{* *} \mathrm{p}<0.01,{ }^{* *} \mathrm{p}<0.001$

When considering users whose scores were lower than 5/10 at baseline (S0), data showed even greater significant positive evolutions: willingness ( 3 to $5, p<0.05$ ); ability ( 3 to $5, p<0.001$ ) and readiness to quit $(3$ to $5, p<0.001)$. Those evolution were present in both premium and free users.

\section{Discussion}

\subsection{Principal findings}

\subsubsection{Adherence and attrition}

From 07/04/2021 to 07/28/2021, a total of 2331 organic users started the preparation program; this means that 2331 users went to an Apple mobile app store, searched for the app, downloaded it, followed the onboarding process, created an account, selected the "prepare for a change" option, before access to the first content' program. We consider this type of user journey as a series of conscious decisions requiring effort and will to quit smoking. Furthermore, considering the number of people who started this program, we can assume a real need. Hence, it is essential to adequately accompany users who wish to quit smoking and make great efforts to seek help.

From the initial sample, only 54 organic users reached the last activity of step 8, which was to set a quit date, meaning that only $2.3 \%$ of the intent-to-treat users completed the program. After completing the program, only 16 users returned to the application, and that was for only one time.

We agree with Katz et al. [27] that is a very low retention rate it highligths the current inability to satisfy expectation of the majority of users seeking to quit. This low retention rate could be explained by three main reasons: expected use of the mobile apps, the target behavior, and the mobile app users' experience. 
1. Expected use of the mobile apps: Attrition rate has been a recognized challenge for general mobile apps, m-health apps and smoking cessation apps tested in a more controlled scenario. In general, $25 \%$ of the most downloaded apps are never used and $38 \%$ of purchased apps are immediately uninstalled [26]. Regarding mhealth apps, the estimated dropout attrition rate is $43 \%$, with higher rates seen in real-world research (2\%) and lower rates in highly supported RCTs (up to 70\%) [27]. In particular, the dropout rate of some smoking cessation apps tested in a more controlled scenario could vary from $19,3 \%$ after 28 days of use [32] to $39,5 \%$ after 87,3 days of use [33].

2. The target behavior : smoking cessation. Based on Prochaska et diClemente's model of the different stages leading to behavior change, the program's target users were those in the preparation stage; this means that the target users intend to act in the next month, but may also be apprehensive about taking action and doubt their ability to succeed. The vast majority of organic users may not be in the preparation phase but the contemplation phase. There is desired change because they are aware of the benefit that this change brings, but they are also aware of the disadvantages involved [34]. Moreover, attrition in smoking cessation interventions of combined behavioral and pharmacological treatment studies ranges from $10.8 \%$ to $77 \%$ [35]. One of the reasons that could have explained the dropout would have been the extrinsic motivational type. However, the results of the analysis did not support this hypothesis.

3. The mobile app users' experience : It could not be only about the theory on which the program is based, but also about the relationship between the user and the interface, as in psychotherapy, where the therapeutic alliance is a critical factor that favors the clients' commitment to their therapeutic process and decreases the dropout rate [36]. In both kinds of relationships, clients evaluate the effortreward ratio to continue engaging in a thérapeutical process.

To examine in more detail when dropouts occur, we compare intra-step and PP interstep dropout rates. Do dropouts occur within a step or at the transition from one step to another? According to the results, there are two significant dropouts and they occur during the transition from one step to the other. The first dropout is between the preliminary step (S0) and the first one (S1). The second dropout is between the second (S2) and third step (S3). Once users start a step, the dropout rate is very low, except S4.

There is a reasonable completion rate in a single step where several activities are proposed, and users are guided (the intra-step completion rate varies between $83 \%$ to 100 $\%)$. On the other hand, when there is a discontinuity, especially in the transition from one step to another, users tend to disengage, as happened between S0 and S1 and during S4, where the user is asked to perform an activity lasting 24 hours, instead of moving directly to the next activity. These activities also imply an active commitment from the user and are not merely passive activity.

From Step 2 to Step 3: There was also a disruption where users were asked to make an in-app purchase to acess to the step 3 content. Any new user could get the 7-day free trial. We assumed that users who started the program mainly were new users, and therefore that they could access the entire program. We did not anticipate the impact of that event. We thought that the vast majority would access the program by subscribing to the offer and then unsubscribing from it. Out of the 2,331 users, only 182 had access to the premium version. One can wonder about the different reasons that users of the free version did not activate the premium. A first hypothesis is that a proportion of the users were not "new" and therefore could not access the free trial. A second hypothesis is that 
the program did not create enough interest to continue in the premium version. A third hypothesis - supported by the results on users' motives for starting a cessation program is that the primary motive for stopping is to save money for free users. We did not anticipate recording the number of people who accessed to the free trial. With such data, we would have a better understanding of the proportion of users who already had access to the application, abandoned it, and then returned to get access to the program. This event will be controlled for the main project by granting access to the premium version to all participants.

In conclusion, the dropout rate can be mitigated by improving the transition from one stage to another to avoid ruptures in the user experience.

\subsubsection{Content clarity and relevance}

Considering that the program content is inspired on evidence-based psychological theories and techniques for behavior change and smoking cessation, we wondered about users' perceptions of how these techniques and principles are adapted in the app. Do they think the program content is clear and relevant to their smoking cessation process? Most users were highly satisfied at the end of each step. On average $85.9 \%(\mathrm{SD}=7.3 \%)$ found the content of each step understandable and relevant. But users' perception of content understandability and relevance did not explain the loss of engagement between both dropouts. For example, $85.8 \%$ of the users find the content understandable and relevant on the introductory step (S0), but only 13\% go through the next step. Likewise, the Step1 content was the one with the higher percentage of users considering it as irrelevant $(27 \%)$. Nonetheless, most of the users who finished Step 1 continued to Step 2 (84\%).

\subsubsection{Readiness to quit}

$60 \%$ of smokers want to quit, but many don't have the proper access to therapeutical interventions [1]. Ambivalence in motivation to change is a very common challenge, and addressing it is part of the therapeutic intervention plan [21, 34].

Engaging in the mobile app preparation program favors positive changes in attitudes toward smoking cessation in users with a low motivation level. Users of the premium version started the program with a higher level of motivation than those on the free version. Those who were less motivated benefited the most from the program in terms of motivational level. They went from a low level of confidence, willingness, and readiness to a medium one (from 3 to 5 in a 1 to 10 EVA scale). These results suggest that the Kwit's preparation program can address ambivalence by increasing willingness to change, self-confidence, and readiness to quit among its users and specially those who felt less prepared.

\subsection{Limitations}

All participants are iOS users. Differences between Android and iOS users were reported in terms of quit attempts and quit date settings [37]. Also, it would have been valuable to have demographic or smoking data on participants before they even started the program, but given the purpose of the study, this information was not necessary. For the study that aims to identify the determinants of regular use of mobile applications to quit smoking, both missing information is planned to be collected. 


\subsection{Future research directions}

Further work is needed to understand the determinants of regular use of mobile health apps. The results of the present study support a hypothesis-generated finding that engagement with the preparation program increases willingness, confidence, and readiness to quit, three key factors for smoking cessation.

The next step includes the implementation and evaluation of program refinements based on current learnings and further evaluation through a quasi-experimental observational study to better understand what enables commitment to use a smoking cessation app, and finally a randomized controlled study to identify the effect of the smoking cessation program on the target behavior. To obtain a user-centered perspective, qualitative studies are needed, and user testing of specific program features should be part of the mobile application development process.

\section{Conclusions}

Overall, Kwit's 9-step preparation program is a functional software ready to assess users' interactions in real-time settings. The study highlights the dropout reality of mobile apps created to help users change complex health behaviors, especially for smoking cessation.

Supplementary Materials: The following are available online at www.mdpi.com/xxx/s1, Figure S1: title, Table S1: title, Video S1: title.

Author Contributions: Conceptualization, L.B.; methodology, L.B and M.E.; validation, L.B., M.E.; formal analysis, M.E.; investigation, L.B.; data curation, M.E.; writing-original draft preparation, L.B.; writing - review and editing, L.B., M.E., L.R.; visualization, L.B and M.E.; supervision, L.R.; Funding acquisition, L.B and L.R. All authors have read and agreed to the published version of the manuscript.

Funding: This research was funded by a financial agreement in the framework of "Territoires de Santé de Demain" by Eurométropole de Strasbourg with a reference number of: TSD-AMI2-2.13, and the $\mathrm{PhD}$ contract is financed by National Association of Research and Technology (ANRT), with a CIFRE reference number: 2019/0078.

Institutional Review Board Statement: This feasibility study is part of a prospective study which aim is to identify key determinants of smoking cessation mobile apps use among smokers seeking to quit. The study was conducted according to the guidelines of the Declaration of Helsinki, European General Data Protection Regulation (RGPD) and approved by the Institutional Review Board the local French Ethical Committee (South-East) on March the 25th, 2021. The Research Identification Number is 2020-A02733-36, and the reference of the committee is CPP 20.10.02.44945.

Informed Consent Statement: No participant is or could be identifiable with the exported data. The Participants of the study consent to share their pseudonymized data by accepting the general terms and conditions and creating their account.

Data Availability Statement: Data is not publicly available due to privacy policy. Authors can share summarized data by e-mail contact at: [2]

Conflicts of Interest: LAB undertakes consultancy and research for a mobile app for smoking cessation (Kwit SAS) in a PhD contract involving the National Association of Research and Technology (ANRT) and the University of Paris-Nanterre. ME is an employee of Kwit SAS. LR have no conflicts to declare.

The funders had no role in the design of the study; in the collection, analyses, or interpretation of data; in the writing of the manuscript, or in the decision to publish the results. 


\section{References}

[1] Organization, W. H. WHO Report on the Global Tobacco Epidemic 2021: Addressing New and Emerging Products: Web Annex VI: Global Tobacco Control Policy Data: Table 6.11; World Health Organization, 2021.

[2] West, R. Tobacco Smoking: Health Impact, Prevalence, Correlates and Interventions. Psychol. Health, 2017, 32 (8), 1018-1036. https://doi.org/10.1080/08870446.2017.1325890.

[3] Sadeghi, M.; Shabib, G.; Masoumi, G.; Amerizadeh, A.; Shahabi, J.; Heidari, R.; Roohafza, H. A Systematic Review and Meta-Analysis on the Prevalence of Smoking Cessation in Cardiovascular Patients After Participating in Cardiac Rehabilitation. Curr. Probl. Cardiol., 2021, 46 (3), 100719. https://doi.org/10.1016/j.cpcardiol.2020.100719.

[4] Patanavanich, R.; Glantz, S. A. Smoking Is Associated with Worse Outcomes of COVID-19 Particularly among Younger Adults: A Systematic Review and Meta-Analysis. BMC Public Health, 2021, 21 (1), 1554. https://doi.org/10.1186/s12889-021-11579-x.

[5] Hartmann-Boyce, J.; Stead, L. F.; Cahill, K.; Lancaster, T. Efficacy of Interventions to Combat Tobacco Addiction: Cochrane Update of 2012 Reviews. Addict. Abingdon Engl., 2013, 108 (10), 1711-1721. https://doi.org/10.1111/add.12291. [6] World Health Organization. WHO report on the global tobacco epidemic, 2017: Monitoring tobacco use and prevention policies.; Geneva, 2017; p 135.

[7] Stead, L. F.; Koilpillai, P.; Fanshawe, T. R.; Lancaster, T. Combined Pharmacotherapy and Behavioural Interventions for Smoking Cessation. Cochrane Database Syst. Rev., 2016, No. 3.

[8] European Commission. Green Paper on mobile health ("mHealth); European Commission: Brussels, 2014 ; p 20.

[9] Perroy, A.-C. La m-santé, à l'ère de la e-santé. Promesses, enjeux et responsabilités. Ann. Pharm. Fr., 2016, 74 (6), 421-430. https://doi.org/10.1016/j.pharma.2016.03.002.

[10] Taylor, G. M. J.; Dalili, M. N.; Semwal, M.; Civljak, M.; Sheikh, A.; Car, J. Internet-Based Interventions for Smoking Cessation. Cochrane Database Syst. Rev., 2017, 9 (9), CD007078. https://doi.org/10.1002/14651858.CD007078.pub5.

[11] Hamari, J.; Koivisto, J. Social Motivations to Use Gamification: An Empirical Study of Gamifying Exercise. 2013. [12] Deterding, S.; Dixon, D.; Khaled, R.; Nacke, L. From Game Design Elements to Gamefulness: Defining Gamification. In Proceedings of the 15th international academic MindTrek conference: Envisioning future media environments; ACM, 2011; pp 9-15.

[13] Sherwani, Y.; Muntasir, M.; Ahmed, M.; El-Hilly, A.; Iqbal, S.; Siddiqui, S.; Al-Fagih, Z.; Eisingerich, A.; Usmani, O. Smoking Cessation Using the Gamification of MHealth Apps: A Longitudinal Qualitative Study. Eur. Respir. J., 2016, 48 (suppl 60), OA3497. https://doi.org/10.1183/13993003.congress-2016.OA3497.

[14] Yıldırım, İ.; Şen, S. The Effects of Gamification on Students' Academic Achievement: A Meta-Analysis Study. Interact. Learn. Environ., 2019, 0 (0), 1-18. https://doi.org/10.1080/10494820.2019.1636089.

[15] Rajani, N. B.; Weth, D.; Mastellos, N.; Filippidis, F. T. Use of Gamification Strategies and Tactics in Mobile Applications for Smoking Cessation: A Review of the UK Mobile App Market. BMJ Open, 2019,9 (6), e027883. https://doi.org/10.1136/bmjopen-2018-027883.

[16] Abu-Dawood, S. The Cognitive and Social Motivational Affordances of Gamification in E-Learning Environment. In 2016 IEEE 16th International Conference on Advanced Learning Technologies (ICALT); IEEE, 2016; pp 373-375.

[17] Rajani, N. B.; Mastellos, N.; Filippidis, F. T. Impact of Gamification on the Self-Efficacy and Motivation to Quit of Smokers: Observational Study of Two Gamified Smoking Cessation Mobile Apps. JMIR Serious Games, 2021, 9 (2), e27290. https://doi.org/10.2196/27290. 
[18] Rajani, N. B.; Mastellos, N.; Filippidis, F. T. Self-Efficacy and Motivation to Quit of Smokers Seeking to Quit: Quantitative Assessment of Smoking Cessation Mobile Apps. JMIR MHealth UHealth, 2021, 9 (4), e25030. https://doi.org/10.2196/25030.

[19] Gwaltney, C. J.; Metrik, J.; Kahler, C. W.; Shiffman, S. Self-Efficacy and Smoking Cessation: A Meta-Analysis. Psychol. Addict. Behav., 2009, 23 (1), 56-66. https://doi.org/10.1037/a0013529.

[20] Ockene, J.; Emmons, K.; Mermelstein, R.; Perkins, K.; Bonollo, D.; Voorhees, C.; Hollis, J. Relapse and Maintenance Issues for Smoking Cessation. Health Psychol. Off. J. Div. Health Psychol. Am. Psychol. Assoc., 2000, 19, 17-31. https://doi.org/10.1037/0278-6133.19.Suppl1.17.

[21] Piñeiro, B.; López-Durán, A.; Del Río, E. F.; Martínez, Ú.; Brandon, T. H.; Becoña, E. Motivation to Quit as a Predictor of Smoking Cessation and Abstinence Maintenance among Treated Spanish Smokers. Addict. Behav., 2016, 53, 40-45. https://doi.org/10.1016/j.addbeh.2015.09.017.

[22] Smit, E. S.; Hoving, C.; Schelleman-Offermans, K.; West, R.; de Vries, H. Predictors of Successful and Unsuccessful Quit Attempts among Smokers Motivated to Quit. Addict. Behav., 2014, 39 (9), 1318-1324. https://doi.org/10.1016/j.addbeh.2014.04.017.

[23] Poushter, J.; Bishop, C.; Chwe, H. Social Media Use Continues to Rise in Developing Countries but Plateaus across Developed Ones. Pew Res. Cent., 2018, 22, 2-19.

[24] Regmi, K.; Kassim, N.; Ahmad, N.; Tuah, N. Effectiveness of Mobile Apps for Smoking Cessation: A Review. Tob Prev Cessat., 2017, 3 (12). https://doi.org/10.18332/tpc/70088.

[25] World Health Organization. Adherence to Long-Term Therapies: Evidence for Action; World Health Organization: Geneva, 2003.

[26] Nielsen, J. Smartphones: So Many Apps, So Much https://www.nielsen.com/us/en/insights/article/2014/smartphones-so-many-apps--so-much-time (accessed Oct 2, 2020). [27] Meyerowitz-Katz, G.; Ravi, S.; Arnolda, L.; Feng, X.; Maberly, G.; Astell-Burt, T. Rates of Attrition and Dropout in App-Based Interventions for Chronic Disease: Systematic Review and Meta-Analysis. J. Med. Internet Res., 2020, 22 (9), e20283. https://doi.org/10.2196/20283.

[28] Horst, M.; Kuttschreuter, M.; Gutteling, J. M. Perceived Usefulness, Personal Experiences, Risk Perception and Trust as Determinants of Adoption of e-Government Services in The Netherlands. Comput. Hum. Behav., 2007, 23 (4), 1838-1852. https://doi.org/10.1016/j.chb.2005.11.003.

[29] Michie, S.; Wood, C. E.; Johnston, M.; Abraham, C.; Francis, J. J.; Hardeman, W. Behaviour Change Techniques: The Development and Evaluation of a Taxonomic Method for Reporting and Describing Behaviour Change Interventions (a Suite of Five Studies Involving Consensus Methods, Randomised Controlled Trials and Analysis of Qualitative Data). Health Technol. Assess. Winch. Engl., 2015, 19 (99), 1-188. https://doi.org/10.3310/hta19990.

[30] Smith, A.; de Salas, K.; Lewis, I.; Schüz, B. Developing Smartphone Apps for Behavioural Studies: The AlcoRisk App Case Study. J. Biomed. Inform., 2017, 72, 108-119. https://doi.org/10.1016/j.jbi.2017.07.007.

[31] Ranganathan, P.; Pramesh, C. S.; Aggarwal, R. Common Pitfalls in Statistical Analysis: Intention-to-Treat versus per-Protocol Analysis. Perspect. Clin. Res., 2016, 7 (3), 144-146. https://doi.org/10.4103/2229-3485.184823.

[32] Ubhi, H. K.; Michie, S.; Kotz, D.; Wong, W. C.; West, R. A Mobile App to Aid Smoking Cessation: Preliminary Evaluation of SmokeFree28. J. Med. Internet Res., 2015, 17 (1), e17. https://doi.org/10.2196/jmir.3479.

[33] Marler, J. D.; Fujii, C. A.; Utley, D. S.; Tesfamariam, L. J.; Galanko, J. A.; Patrick, H. Initial Assessment of a Comprehensive Digital Smoking Cessation Program That Incorporates a Mobile App, Breath Sensor, and Coaching: Cohort Study. JMIR MHealth UHealth, 2019, 7 (2), e12609. https://doi.org/10.2196/12609.

[34] Prochaska, J. O.; Prochaska, J. M. Changing to Thrive: Using the Stages of Change to Overcome the Top Threats to Your 
Health and Happiness; Simon and Schuster, 2016.

[35] Belita, E.; Sidani, S. Attrition in Smoking Cessation Intervention Studies: A Systematic Review. Can. J. Nurs. Res. Rev. Can. Rech. En Sci. Infirm., 2015, 47 (4), 21-40. https://doi.org/10.1177/084456211504700402.

[36] Roos, J.; Werbart, A. Therapist and Relationship Factors Influencing Dropout from Individual Psychotherapy: A Literature Review. Psychother. Res., 2013, 23 (4), 394-418. https://doi.org/10.1080/10503307.2013.775528.

[37] Ubhi, H. K.; Kotz, D.; Michie, S.; van Schayck, O. C. P.; West, R. A Comparison of the Characteristics of IOS and Android Users of a Smoking Cessation App. Transl. Behav. Med., 2017, 7 (2), 166-171. https://doi.org/10.1007/s13142-0160455-z. 